\title{
B2 1144+35B, a giant low power radio galaxy with superluminal motion
}

\section{Orientation and evidence for recurrent activity}

\author{
G. Giovannini ${ }^{1,2}$, M. Giroletti ${ }^{2}$, and G. B. Taylor ${ }^{3}$ \\ ${ }^{1}$ Dipartimento di Astronomia, Universita' di Bologna, via Ranzani 1, 40127 Bologna, Italy \\ e-mail: ggiovann@ira.inaf.it \\ 2 Istituto di Radioastronomia - INAF, via Gobetti 101, 40129 Bologna, Italy \\ 3 Department of Physics and Astronomy, University of New Mexico, Albuquerque, NM 87131, USA
}

Received 11 June 2007 / Accepted 3 August 2007

\section{ABSTRACT}

\begin{abstract}
Aims. The goal of this work is a detailed study of the nearby, low power radio galaxy B2 1144+35B.
Methods. For this purpose, we performed new Very Long Baseline Array (VLBA) and Very Large Array (VLA) observations.

Results. This source has several properties (bright, nearby, large range of spatial structures, visible counterjet, etc.) that make it an excellent astrophysical laboratory for the study of the evolution of radio jets. Here we report the detection of motion in the counterjet at $0.23 \pm 0.07 \mathrm{c}$, which allows us to estimate the orientation of the parsec-scale jet at $33^{\circ} \pm 7^{\circ}$ from the line of sight, with an intrinsic velocity of $\left(0.94_{-0.11}^{+0.06}\right) \mathrm{c}$. We also report on a brightening of the core at high frequencies which we suggest could be the result of a new component emerging from the core. High dynamic range VLBA observations at $5 \mathrm{GHz}$ reveal, for the first time, extended emission connecting the core with the bright complex of emission that dominates the flux density of the parsec scale structure at frequencies below $20 \mathrm{GHz}$. The evolution of this bright complex is considered and its slow decline in flux density is interpreted as the result of an interaction with the interstellar medium of the host galaxy.
\end{abstract}

Key words. galaxies: active - galaxies: jets - galaxies: nuclei - galaxies: individual: B2 1144+35B

\section{Introduction}

The low power radio galaxy B2 1144+35B (hereafter referred to as $1144+35)$ is identified with a faint $\left(m_{\mathrm{pg}}=15.7\right)$ Zwicky galaxy (ZW186.48) in a medium-compact galaxy cluster at a redshift of 0.0630 .

In an optical study of bright, flat-spectrum radio sources, Marcha et al. (1996) classify $1144+35$ as a BL Lac candidate even though its spectrum shows $\mathrm{H} \alpha$ and [NII] emission lines (Colla et al. 1975; Morganti et al. 1992). From a comparison between the measured line equivalent width and the line contrast, Marcha et al. (1996) suggest that $1144+35$ could be a diluted BL Lac. 1144+35 has also been included in an imaging and spectroscopic survey of low and intermediate power radio galaxies (Ebneter 1989). A CCD residual image shows a very definite arc of dust in the nuclear region of the host galaxy.

From the radio point of view, $1144+35$ has a peculiar structure as discussed in detail by Giovannini et al. (1999). It is characterized by a large scale FR-I radio structure and Machalski (1998) classifies it among the giant radio galaxies. The total angular size is $\sim 13^{\prime}$ corresponding to a projected linear size of $\sim 0.9 \mathrm{Mpc}$. The total radio power at $1.4 \mathrm{GHz}$ is $6.3 \times 10^{24} \mathrm{~W} \mathrm{~Hz}^{-1}$. Despite its large size, the kiloparsec scale radio structure is core dominated with a short, bright two-sided jet. The arcsecond core exhibits long-term flux density variability. Its flux density showed a large increase from 1974 to 1980 followed by a smooth increase until 1992. From 1992 until 1999 the arcsecond core flux density has been decreasing (Giovannini et al. 1999).
The first VLBI observations of this source were carried out by Giovannini et al. (1990), and successive VLA, MERLIN and VLBI data (Giovannini et al. 1999) reveal complex structure over a broad range of physical scales $(1 \mathrm{pc}-1 \mathrm{Mpc})$. The bright arcsecond scale core is resolved at milliarcsecond resolution into a nuclear source, a main jet with an apparent superluminal velocity, and a faint counter-jet (cj).

We present here new multi-frequency VLBA observations (Sect. 2) to study the source evolution, proper motion, flux density variability, and radio spectral index (Sect. 3). Moreover we present flux density measurements of the arcsecond core in order to compare its variability with the parsec scale source evolution. Results are discussed in Sect. 4, and conclusions are given in Sect. 5 .

Assuming $H_{0}=70 \mathrm{~km} \mathrm{~s}^{-1} \mathrm{Mpc}^{-1}, \Omega_{\mathrm{m}}=0.3$ and $\Omega_{\Lambda}=$ 0.7 , the luminosity distance $\left(D_{1}\right)$ for $1144+35$ is $282.7 \mathrm{Mpc}$, the angular distance $\left(D_{\mathrm{a}}\right)$ is $250.2 \mathrm{Mpc}$ with a conversion factor $=1.21 \mathrm{kpc} /{ }^{\prime \prime}$.

\section{Observations and data reduction}

\subsection{VLBI data}

New observations have been obtained with the VLBA of the NRAO $^{1}$ on February 17, 2002 at 5 and $8.4 \mathrm{GHz}$ and on

1 The National Radio Astronomy Observatory is operated by Associated Universities, Inc., under cooperative agreement with the National Science Foundation. 
Table 1. Arcsecond core flux densities.

\begin{tabular}{ccccccc}
\hline \hline $\begin{array}{c}\text { Epoch } \\
\text { mm-yy }\end{array}$ & $\begin{array}{c}\text { Flux (mJy) } \\
1.4 \mathrm{GHz}\end{array}$ & $\begin{array}{c}\text { Flux (mJy) } \\
5.0 \mathrm{GHz}\end{array}$ & $\begin{array}{c}\text { Flux (mJy) } \\
8.3 \mathrm{GHz}\end{array}$ & $\begin{array}{c}\text { Flux (mJy) } \\
15 \mathrm{GHz}\end{array}$ & $\begin{array}{c}\text { Flux (mJy) } \\
22 \mathrm{GHz}\end{array}$ & $\begin{array}{c}\text { Flux (mJy) } \\
43 \mathrm{GHz}\end{array}$ \\
\hline $03-02$ & 399 & 330 & 263 & 226 & 200 & 146 \\
$06-06$ & 338 & 243 & 222 & 204 & 193 & 153 \\
\hline
\end{tabular}
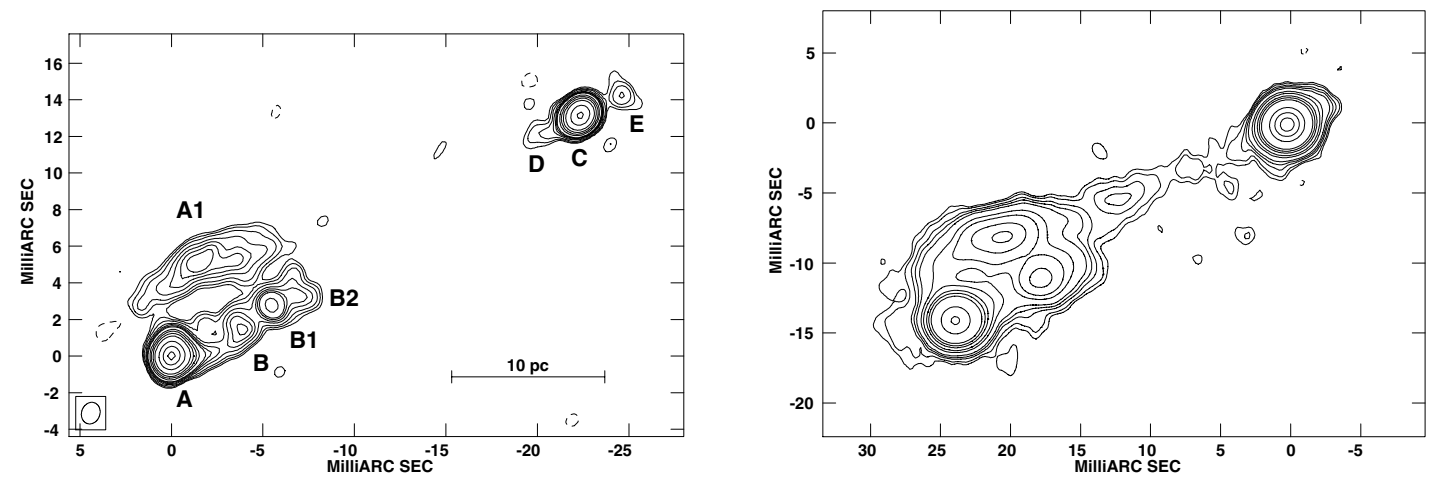

Fig. 1. VLBI images of $1144+35$ at $8.4 \mathrm{GHz}$. Left: 2002 epoch; the HPBW is $1.2 \times 1.0$ mas in PA $-20^{\circ}$; the noise level is $0.1 \mathrm{mJy} / \mathrm{beam}$. Contours are: $-0.50 .50 .711 .522 .53571015305070 \mathrm{mJy} / \mathrm{beam}$. Letters indicate different components as discussed in the text. Right: 2005 epoch; the HPBW is $2 \times 2$ mas; the noise level is $0.05 \mathrm{mJy} /$ beam. Contours are drawn at: $-0.30 .20 .30 .50 .71235710305070 \mathrm{mJy} / \mathrm{beam}$.

September 22, 2005 at 1.5, 5, and $8.4 \mathrm{GHz}$. Observations were carried out, switching often between different frequencies in order to obtain good and uniform $(u, v)$ coverage. The data were correlated in Socorro with the VLBA correlator. Amplitude calibration was performed using observations of the system temperature and antenna gains in the standard way. Data were globally fringe-fitted and calibrated in AIPS. We made several iterations of phase self-calibration, followed by a final phase and gain selfcalibration when necessary to produce the final image.

Observations at $8.4 \mathrm{GHz}$ (2005 epoch) were performed in a phase-referencing mode in order to obtain a good core position useful for future studies of absolute motion. We used $\mathrm{J} 1130+3815$ as primary calibrator, and $\mathrm{J} 1130+3031$, $\mathrm{J} 1214+3309$ as secondary calibrators.

\subsection{VLA data}

We obtained a $\sim 2 \mathrm{~h}$ observation with the VLA (NRAO) in the A (March 2002) and A/B configuration (June 2006) to investigate the flux density variability of the arcsecond scale core. The data have been calibrated in the standard way using the NRAO AIPS package and imaged using the task IMAGR. In these images due to the lack of short spacings and the short integration time, only the unresolved arcsecond core is visible and therefore we make use of these data only to discuss the variability of the arcsecond core. Observations were performed at 6 different frequencies and the core flux density for each is reported in Table 1 . The core flux density was derived by fitting a Gaussian to the nuclear source in the image plane.

\section{Results}

\subsection{Parsec scale morphology, variability, and spectral indices}

The new observations confirm the general structure reported in Giovannini et al. (1999). We label the different components as shown in Fig. 1, in accordance with Giovannini et al. (1999).

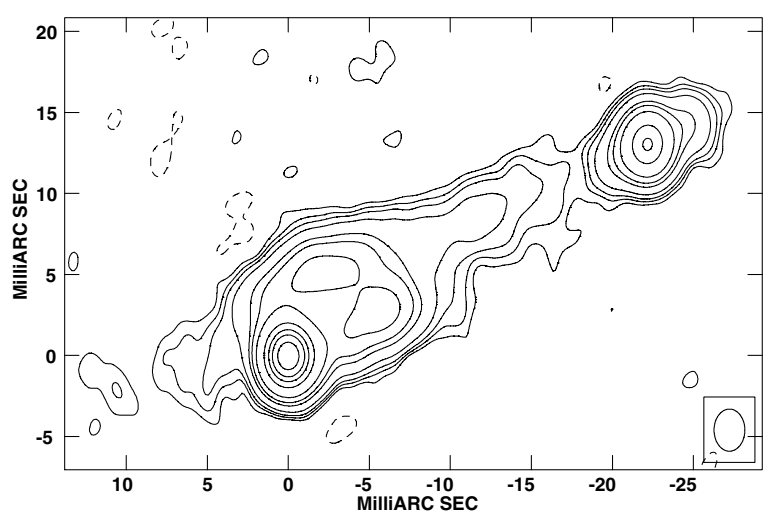

Fig. 2. VLBI image of $1144+35$ at $5 \mathrm{GHz}$ (2005 epoch). The HPBW is $2.6 \times 1.9 \mathrm{mas}$ in PA $0^{\circ}$; the noise level is $0.06 \mathrm{mJy} / \mathrm{beam}$. Contours are drawn at: $-0.150 .150 .30 .513510305070100 \mathrm{mJy} / \mathrm{beam}$.

The parsec scale structure at 5 and $8 \mathrm{GHz}$ (Figs. 1 and 2) is resolved in a nuclear source (C) with a short jet (D) and cj structure (E). The core position from the phase reference data is: RA $=11$ $4722.12902 \mathrm{Dec}=+350107.5350(\mathrm{~J} 2000)$. At about 25 mas east of the core we detect an extended jet like structure (A and B components) which dominates the flux density in the VLBI images. This jet structure is extended, clearly limb-brightened, and connected to the core by a low-brightness emission. The opening angle is $\sim 10^{\circ}$, in agreement with the value found by Giovannini et al. (1999). The shape of this extended feature is similar in different epoch of observations. In particular the high-resolution images at $8.4 \mathrm{GHz}$ confirm that component $\mathrm{A} 1$ is resolved into an extended complex structure aligned with components C, D, and E. Furthermore this complex structure curves in the direction of component A, suggesting a possible jet recollimation, most evident in the last epoch. Moreover a fairly compact component B2, not present in 1997 images, has emerged just west 


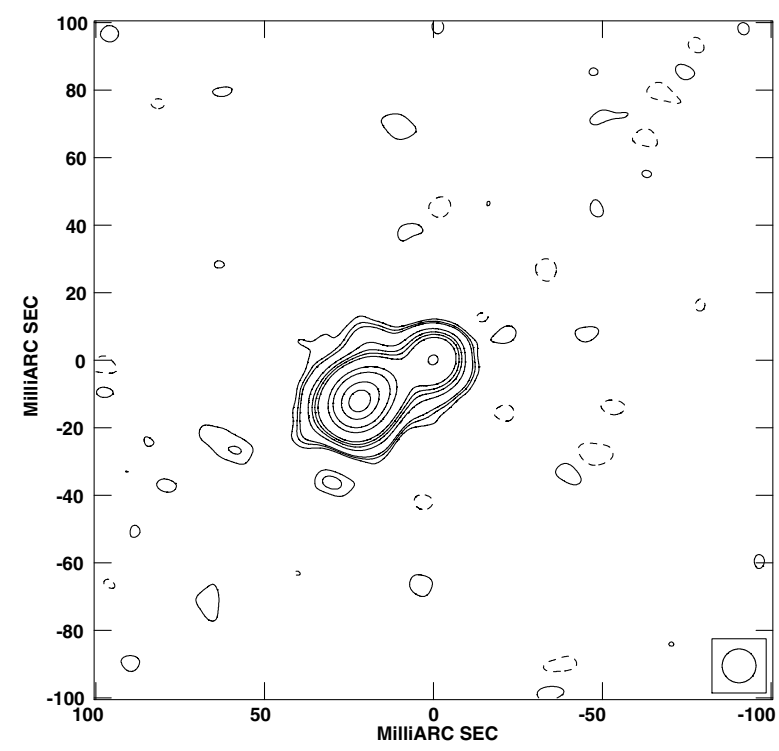

Fig. 3. VLBI image of $1144+35$ at $1.5 \mathrm{GHz}$ (2005 epoch). The HPBW is $10 \times 10 \mathrm{mas}$; the noise level is $0.14 \mathrm{mJy} / \mathrm{beam}$. Contours are drawn at: $-0.50 .40 .71357103050100150 \mathrm{mJy} /$ beam.

of component B1. The flux density of compact sub-structures is not constant.

At $5 \mathrm{GHz}$ and in the low-resolution $8.4 \mathrm{GHz}$ images, for the first time, the connection between the two-sided core region and the distant jet structure is readily visible confirming the peculiar alignment between components $\mathrm{A} 1, \mathrm{D}, \mathrm{C}$, and $\mathrm{E}$ while components $\mathrm{A}, \mathrm{B}, \mathrm{B} 1$, and $\mathrm{B} 2$ are slightly off axis. A low-brightness emission in front of component $\mathrm{A}$ is present, probably connected to component A1.

At lower frequency (1.4 GHz, Fig. 3) no low brightness emission is visible at larger distances from the core. The faint ( $\sim 1 \mathrm{mJy} /$ beam $)$ extended feature seen in 1995 observations at $\sim 90$ mas from the core (Giovannini et al. 1999), aligned with the $A+B$ structure, if real, could have been missed because of lower brightness due to expansion. The extension just in front at the compact A component is confirmed. The jet is transversally resolved suggesting the presence of a low brightness steep spectrum emission surrounding the brighest jet regions visible in high resolution maps at 5 and $8.4 \mathrm{GHz}$.

In Table 2 we report the measured flux density at different epochs and frequencies for each component. Components are named according to Fig. 1. JT is the total flux density of the main jet (A, A1, B, B1, B2 components). It can be slightly higher than the sum of different components because of low surface flux density in between the single substructures. The flux density variability is shown in Fig. 4 for the main components. We show only $8.4 \mathrm{GHz}$ data, since at this frequency better statistics are available, and we have the high angular scale images necessary to separate the various components.

We derived the spectrum of individual components using data at the 2005.72 epoch when three different frequencies were available. We confirm the results published in Giovannini et al. (1999): i) component $\mathrm{C}$ is the only self-absorbed component with the self-absorption frequency $\gtrsim 8.4 \mathrm{GHz}\left(\alpha_{1.4}^{8.4}=\right.$ $-0.61 \pm 0.03$, assuming $\left.\mathrm{S}(v) \propto v^{-\alpha}\right)$; ii) the spectral shape of component $\mathrm{A}$, despite the large variation in flux density, did not change: $\alpha_{1.4}^{5.0}=0.51 \pm 0.02$ with a high frequency flattening
Table 2. Flux densities at various epochs of VLBI components.

\begin{tabular}{ccccc}
\hline \hline Component & $\begin{array}{c}\text { Epoch } \\
\text { yr }\end{array}$ & $\begin{array}{c}8.4 \mathrm{GHz} \\
\mathrm{mJy}\end{array}$ & $\begin{array}{c}5 \mathrm{GHz} \\
\mathrm{mJy}\end{array}$ & $\begin{array}{c}1.4 \mathrm{GHz} \\
\mathrm{mJy}\end{array}$ \\
\hline A & 1995.22 & 255 & - & - \\
A & 1995.90 & 224 & - & - \\
A & 1997.74 & 184 & - & - \\
A & 2002.13 & 103 & 147 & - \\
A & 2005.72 & 63 & 68 & 130 \\
B & 1995.22 & 28.7 & - & - \\
B & 1995.90 & 37.0 & - & - \\
B & 1997.74 & 21.8 & - & - \\
B & 2002.13 & 11.9 & - & - \\
B & 2005.72 & 2.9 & 6.2 & - \\
C & 1995.22 & 59.7 & - & - \\
C & 1995.90 & 62.6 & - & - \\
C & 1997.74 & 64.9 & - & - \\
C & 2002.13 & 53.7 & 50.1 & - \\
C & 2005.72 & 74.0 & 43.4 & 24.6 \\
A1 & 1995.22 & 41.5 & - & - \\
A1 & 1995.90 & 34.9 & - & - \\
A1 & 1997.74 & 43.7 & - & - \\
A1 & 2002.13 & 27.5 & 42.3 & - \\
A1 & 2005.72 & 29.3 & 32.5 & - \\
B1 & 2002.13 & 9.6 & - & - \\
B1 & 2005.72 & 2.0 & 6.7 & - \\
B2 & 2002.13 & 5.6 & - & - \\
B2 & 2005.72 & 7.5 & 6.2 & - \\
JT & 2002.13 & 164 & 232 & - \\
JT & 2005.72 & 115 & 125 & 269 \\
\hline & & & & \\
\hline
\end{tabular}

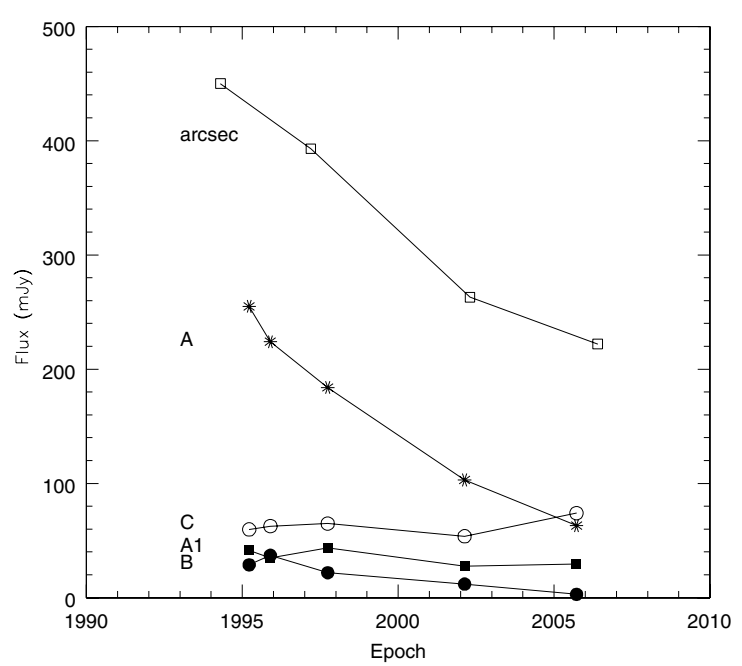

Fig. 4. Flux density measures at $8.4 \mathrm{GHz}$ at different epochs of the arcsecond core and of parsec scale components named according to Fig. 1. Connecting lines are drawn for display purpose. Components B1 and B2 are omitted for clarity and because of their low flux density. Flux density errors are $2 \%$ or $0.1 \mathrm{mJy}$ for fainter components.

$\left(\alpha_{5.0}^{8.4}=0.15 \pm 0.04\right)$ in agreement with the compact structure of this component; iii) other components show a moderately steep spectrum. The average spectrum of the whole strong jet component is $\alpha_{1.4}^{8.4} \sim 0.5$.

Based on its spectral properties and compactness we confirm component $\mathrm{C}$ as the center of activity for $1144+35 \mathrm{~B}$.

Using 5 and $8.4 \mathrm{GHz}$ data obtained at the same epoch we produced images with the same $(u, v)$ range, cellsize and angular resolution. We used these data to produce a spectral index image. The spectral index image confirms the properties of single components as discussed above, moreover it shows that the short 
Table 3. Distances from the core at different epochs.

\begin{tabular}{ccccc}
\hline \hline $\begin{array}{c}\text { Epoch } \\
\text { year }\end{array}$ & $\begin{array}{c}\text { E } \\
\text { mas }\end{array}$ & $\begin{array}{c}\text { A } \\
\text { mas }\end{array}$ & $\begin{array}{c}\text { B } \\
\text { mas }\end{array}$ & $\begin{array}{c}\text { Frequency } \\
\text { GHz }\end{array}$ \\
\hline 1990.89 & - & 20.2 & 16.4 & 5.0 \\
1993.44 & - & 21.4 & 18.0 & 5.0 \\
1995.22 & 2.1 & 22.5 & 18.6 & 8.4 \\
1995.90 & 2.2 & 22.8 & 18.9 & 8.4 \\
1997.74 & 2.4 & 23.8 & 19.8 & 8.4 \\
2002.13 & 2.6 & 26.0 & 22.0 & 8.4 \\
2005.72 & 2.7 & 27.6 & 24.4 & 8.4 \\
\hline
\end{tabular}

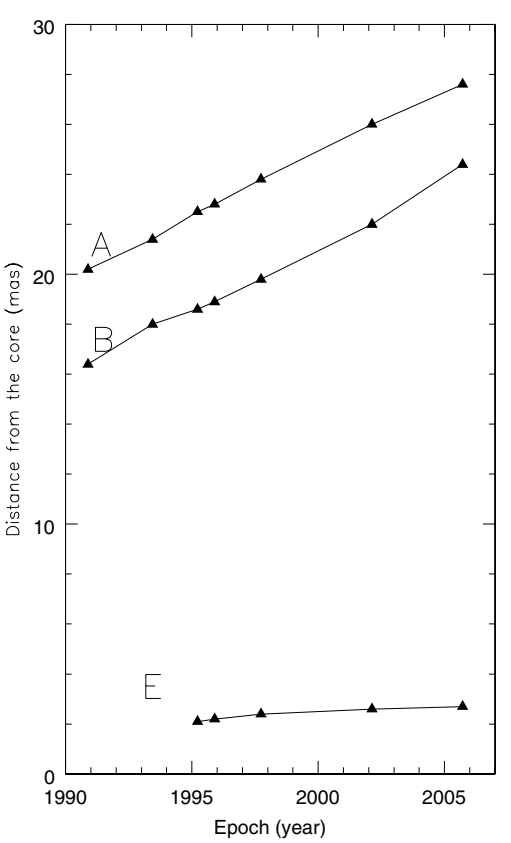

Fig. 5. Distance of A, B, and E components from the parsec scale core (C) at different epochs. Connecting lines are for display only.

jet and cj components (D and E) have a steep spectrum $(\alpha \sim 1.0)$ and that also the low brightness structure connecting D to A1 is very steep ( $\alpha$ in the range 1.1-1.6).

\subsection{Proper motion}

We used the observations made at different epochs to measure the apparent proper motion of components A, B, and E with respect to the core component $\mathrm{C}$. We note that new observations confirm the result discussed in Giovannini et al. (1999) that the relative positions of main jet sub-structures appear constant in time. Also the radio emission centroid of component A1 in different epoch images is always in between the A and B components, suggesting a similar apparent velocity for all the bright jet structure. The position of the short jet $\mathrm{D}$ is poorly defined being smooth with no substructure, therefore it is not possible to measure a reliable proper motion for it.

In Table 3 and Fig. 5 we show the distance of components A, $\mathrm{B}$, and $\mathrm{E}$ from $\mathrm{C}$ at different epochs. For component $\mathrm{E}$ we use only high resolution $8.4 \mathrm{GHz}$ data. By considering all observations to date we measure for the first time a proper motion of the $\mathrm{cj}(\mathrm{E})$ structure in agreement with the previous upper limit: $\beta_{\text {acj }}=0.23 \pm 0.07$, while we confirm $\beta_{\text {aj }}=1.92 \pm 0.05$ for components $\mathrm{A}$ and $\mathrm{B}$ (note the different cosmology used here with respect to previous papers).

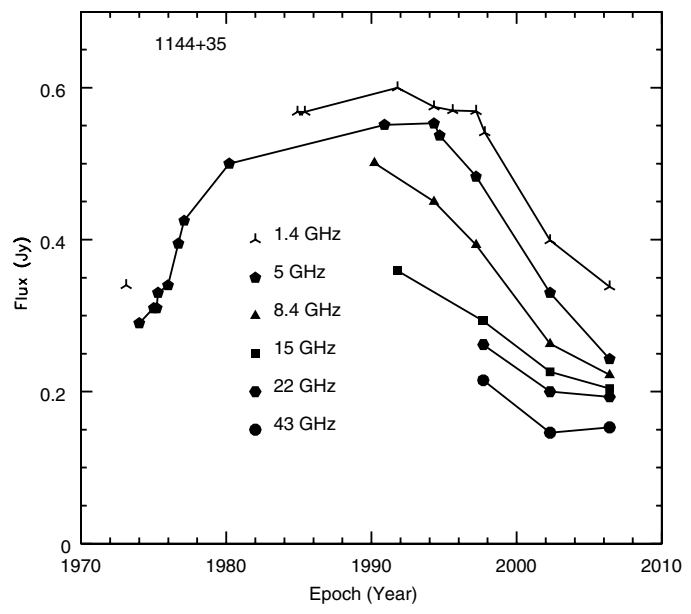

Fig. 6. Core flux density at arcsecond resolution, at different epochs and frequencies. Lines connecting points are for display only.

\subsection{The arcsecond core}

The arcsecond core of $1144+35$ is the dominant feature of the radio emission from this galaxy and has long been known to be variable (Ekers et al. 1983). The arcsecond core flux densities available in the literature have been presented in Giovannini et al. (1999). We add here new recent measurements (Table 1). In Fig. 6 we present the plot of the arcsecond core flux density at different epochs and frequencies.

We derive the core spectral index by comparing observations at the same time or very near in time. We note that from 1990 until 2002 we have a flat spectrum on the lower frequency range $\left(\alpha_{1.4}^{5.0}\right)$ which however is steepening in time $(0.10$ in $1990,0.22$ in 2002). In the higher-frequency range (5 to 15 or $43 \mathrm{GHz}$ ) the spectral index is constant in time and slightly steeper: $\alpha_{5.0}^{43}=0.38$. In the 2006 epoch we have a significant change with a flattening in the higher frequency range: $\left(\alpha_{1.4}^{5.0}=0.26\right.$ and $\left.\alpha_{5.0}^{43}=0.21\right)$. Uncertainties in the above spectral index values are $\sim 0.03$.

\section{Discussion}

\subsection{Jet orientation and velocity}

From the measured apparent proper motion of the parsec scale jet and cj, assuming intrinsic symmetry, we can derive $\beta \cos \theta$ for this parsec scale jet independently of the Hubble constant following Mirabel \& Rodriguez (1994):

$\left(\beta_{\mathrm{aj}}-\beta_{\mathrm{acj}}\right) /\left(\beta_{\mathrm{aj}}+\beta_{\mathrm{acj}}\right)=\beta \cos \theta$

We find:

$\beta \cos \theta=0.79 \pm 0.07$.

Since we know the source $D_{\mathrm{a}}$ in the present cosmology we can also derive the intrinsic jet orientation (Mirabel \& Rodriguez 1994):

$\tan \theta=\left(2 D_{\mathrm{a}} \mu_{\mathrm{j}} \mu_{\mathrm{cj}}\right) /\left(c\left(\mu_{\mathrm{j}}-\mu_{\mathrm{cj}}\right)\right)$

where $\mu_{\mathrm{j}}$ and $\mu_{\mathrm{cj}}$ are respectively the jet and cj angular velocity from proper motion measures.

We find $\theta=33^{\circ} \pm 7^{\circ}$ and therefore $\beta=0.94_{-0.11}^{+0.06}$ is the intrinsic value of the jet velocity in $\mathrm{c}$ units ${ }^{2}$. These values agree with

\footnotetext{
${ }^{2}$ Note that we did not assume an error for the Angular Distance.
} 
the measured jet-cj arm ratio $(\sim 10)$ which implies (Giovannini et al. 1998; Taylor \& Vermeulen 1997):

$\beta \cos \theta \sim 0.8$.

Therefore the pattern jet velocity from proper motion is in agreement with the jet bulk velocity derived from the jet-cj arm ratio. The Lorentz factor $\gamma$ is 2.9 and the Doppler factor $\delta$ is $\sim 1.6$.

\subsection{Parsec scale jet morphology}

In light of previous results and in agreement with the discussion given in Giovannini et al. (1999), the limb-brightened jet morphology can be explained by a transversal velocity structure. The observed brightness of the external jet regions is amplified (the Doppler factor is $\delta \sim 1.6)$. If we assume that the inner jet spine is moving at higher velocity (e.g. $\beta \sim 0.998$ ), the spine Doppler factor will be $\delta \sim 0.39$, therefore the observed brightness of the inner spine is dimmed and the jet will appear limb-brightened. A similar structure was found in the BL Lac object Markarian 501 (see Giroletti et al. 2004).

In this scenario the gap between the core and the A+B complex is explained by the low value $(<1)$ of the Doppler factor before the velocity decrease of the outer sheath. The jet substructures are regions with a strong interaction between the jet and the surrounding medium, with a jet velocity decrease and a higher observed brightness. The large asymmetry between A1 and $\mathrm{A}+\mathrm{B}+\mathrm{B} 1+\mathrm{B} 2$ side could reflect a different interaction between the jet and an inhomogeneous ISM (see Giovannini et al. 1999, for a more detailed discussion).

We note that a jet velocity structure is readily detected only in sources in a narrow range of orientation with respect to the line of sight: in sources near the plane of sky only the external shear is visible, while in sources at a small angle with respect to the line of sight, the fast spine is highly boosted and dominates the radio images.

\subsection{Flux density variability}

The arcsecond core flux density of $1144+35$ shows a slow, but well defined variability (Fig. 6). The core flux density increases from 1974 to $\sim 1994$ and decreases from 1994 to 2002 at all frequencies. From a comparison of the flux density variability of arcsecond and VLBI components (see Fig. 4 and Tables 1 and 2), it is clear that the flux density decrease is not due to the VLBI core but to changes in the compact jet component $\mathrm{A}$. The reason of the strong flux density decrease of component $\mathrm{A}$ is not clear:

- The decrease cannot be due to adiabatic expansion since in all epochs this component is slightly resolved in images at $8.4 \mathrm{GHz}$ and we did not measure any increase of its size;

- we exclude a flux density decrease because of radiation losses related to the aging of relativistic particles, because of the too short time scale and the constant spectrum at different epochs (moreover the flux density decrease is present also at $1.4 \mathrm{GHz}$ );

- the decrease cannot be due to a change of the Doppler factor because of a different orientation angle with respect to the line of sight, or a jet velocity decrease since the proper motion of the whole bright jet structure is regular and constant in time.

It could be due to a change of the external medium: component $\mathrm{A}$ being the most distant component of the jet substructure could be interacting with a dense cloud and local reaceleration processes have increased its brightness. In this scenario, around 1995 the A component emerged from a cloud and its brightness started to decrease. A dynamic interaction of the whole jet structure ( $\mathrm{A}+\mathrm{B}$ components $)$ is supported also by the small changes in its morphology such as the presence of a new sub-component (B2) with respect to previous images. This is a speculative, if real we should see a change in the proper motion velocity: slower before (during the interaction with the cloud) and faster after the interaction. Unfortunately we started to monitor the parsec scale structure when the flux density was already decreasing (after the cloud interaction) so that we cannot yet put this model to the test.

We note that in the 2002-2006 period the arcsecond core flux density decrease stopped in the high frequency range (we have a flux density increase at $43 \mathrm{GHz}$ and a constant value at $22 \mathrm{GHz}$ ). This change in the flux density variability is not due to the A component (see Fig. 4), but to an increase in the VLBI core flux density, which became in 2006 the strongest component on the VLBI scale. A possible interpretation of this result is that the core $\mathrm{C}$ began a new active phase with the emission of a new component not yet visible in our images because of its small size and short distance from the core. This component is still self absorbed at frequencies lower than $22 \mathrm{GHz}$ and we find indication of its existence only at 22 and mostly at $43 \mathrm{GHz}$. Therefore only the arcsecond core flux density measured at high frequency is at present affected by this new component. Assuming a selfabsorption frequency above $>8.4 \mathrm{GHz}$, and equipartition conditions, the size of this new component is $<0.03$ mas. If this component is moving at the same expanding velocity of the jet, we should start to resolve it in VLBI images at $8.4 \mathrm{GHz}$ in $\gtrsim 2$ years.

\subsection{Source evolution}

The large scale structure shows a clear discontinuity between the extended relaxed lobes and the high brightness arcsecond core and jets. These observational data suggest different phases in the life of this radio source: the extended structure is a relic emission with an age in the range 5 to $9 \times 10^{7}$ yr as estimated in Giovannini et al. (1999), while the emission on the arcsecond-scale has a shorter dynamical age: assuming an average velocity of $\gtrsim 0.02 \mathrm{c}$ for the arcsecond structure we derive an age of $\lesssim 1.0 \times 10^{7} \mathrm{yr}$, taking into account projection effects.

We note also that the parsec scale jet emission does not decrease smoothly in our VLBI maps. The main parsec scale jet appears to stop at the end of the A component at 30 mas from the core. Assuming a constant velocity (see Fig. 5) the main VLBI jet structure was emitted from the core a few decades ago, circa 1950. Moreover we see from VLBI images the main component $\mathrm{A}$ is followed by a complex bright structure with many subcomponents suggesting that the source activity related to the emission of the A component lasted about 10-20 years with an almost continuous ejection. Presumbably source activity and jet production was also present in epochs before 1950, since there are evidences from MERLIN data that a low level flux density emission is present at larger distances from the core, in the same position angle. Moreover the short arcsecond-scale jet shows a high brightness well defined structure. We can suppose that multiple activity phases happened in the last $10^{7} \mathrm{yr}$. In this scenario we can add the evidence discussed in the previous sub-section that a new component was emitted in $\sim 2002$ and should be visible at $8.4 \mathrm{GHz}$ in a couple of years, giving us the opportunity to study its evolution in connection with the arcsecond-scale flux density variability. 
Finally we note that the new data do not add any information to understanding the reason for the asymmetry between the A1 and $\mathrm{A}+\mathrm{B}$ jet structures. This remains a puzzle.

\section{Conclusions}

New observations discussed here confirm the complex parsecscale structure of the source $1144+35$. This parsec-scale structure is well-defined and we can easily follow it in different epochs. However some changes are evident, in particular the flux density decrease of the A component and the presence of a new fairly compact component B2 in the main jet structure, suggesting a dynamic interaction with the surrounding medium and possible local particle reacceleration by turbulence. Such interactions may trigger star formation (Croft et al. 2006), and influence the evolution of the host galaxy. Feedback mechanisms between the growth of the central black hole and the galaxy evolution have been shown to be important in large elliptical galaxies in clusters (Allen et al. 2006), and could be important in lower luminosity systems as well.

A few peculiarities have not yet been understood: 1) high sensitivity images reveal the connection between the core $C$ and the bright jet substructure confirming the peculiar alignment between components $\mathrm{A} 1, \mathrm{D}, \mathrm{C}$, and $\mathrm{E}$ while other bright components (A, B, B1, B2) are slightly off axis. 2) The low brightness emission in front of component $\mathrm{A}$ is in contrast with our favored scenario in which the bright spot $\mathrm{A}$ is the working surface of the jet impinging on the surrounding medium. 3) The evident asymmetry between components $\mathrm{A} 1$ and the $\mathrm{A}, \mathrm{B}, \mathrm{B} 1$, and $\mathrm{B} 2$ complex, possibly due to a different interaction with the ISM, implies different properties in the ISM on the parsec-scale. 4) Investigation into the low brightness extended emission surrounding the bright jet components will require higher sensitivity data before it can be properly discussed.

The study of the flux density variability in this source is important to understand the small-scale structures. The recent increase in the core (component $\mathrm{C}$ ) flux density at high frequency is interpreted as evidence of a new jet component expelled from the core a few years ago and now moving along the jet. Further along the jet, the large flux density decrease of the arcsecond core from 1992 to 2002 is due mainly to the jet component A, but the physical mechanism is still unknown.

A spectral index study confirms the core identification with the only self-absorbed component and shows that compact subcomponents have a flatter spectrum with respect to extended low brightness regions.

The proper motion of jet components is regular and constant in time. The detection of a cj proper motion allows us to derive the intrinsic jet velocity and orientation with respect to the line of sight. These results are in agreement with the estimate of the bulk jet velocity derived from the jet to cj arm ratio.

The radio morphology of this source shows clear discontinuities at different linear scales suggesting a continuous activity but with high and low level periods. The connection between this continuous, but not constant, radio activity of the central AGN with the evolution of the parent galaxy is not yet understood.

The radio galaxy $1144+35$ is an interesting astrophysical laboratory to study parsec scale jet properties and evolution and their connection with the kiloparsec scale structure, because of its low redshift, complex but well studied structure, constant but slow variability in flux density and morphology, a well detected jet and counter-jet proper motion, and a large amount of available data.

If we compare this source with other low-power radio galaxies, we find that the measured jet velocity and orientation agrees with unified model predictions and with general parameters derived from statistical studies (e.g. see Giovannini et al. 2001). $1144+35$ is one of the few sources where the jet velocity from proper motion is in agreement with the jet velocity from the jet-cj arm ratio. However some properties of this source are quite peculiar and not yet understood as discussed before. In particular the level of radio activity in this source is clearly not constant in time. Hints of recurrent activity have been found in other low-power radio galaxies, but sufficiently detailed studies for a comparison to $1144+35$ are lacking. We can conclude that, while some general properties of low-power radio sources are at present well-established (e.g. existence of relativistic jets on the parsec scale, jet velocity decrease with core distance, connection between $\mathrm{pc}$ and $\mathrm{kpc}$ scale jets), properties of restarted radio sources are still poorly known and require deep and detailed studies.

Acknowledgements. We thank Dr. Luigina Feretti for useful discussions and suggestions, and an anonymous Referee for useful and important comments. This work was partially supported by the Italian Ministry for University and Research (MIUR) and by the National Institute for Astrophysics (INAF).

\section{References}

Allen, S. W., Dunn, R. J. H., Fabian, A. C., Taylor, G. B., \& Reynolds, C. S. 2006, MNRAS, 372, 21

Colla, G., Fanti, C., Fanti, R., et al. 1975, A\&AS, 20, 1

Croft, S., van Breugel, W., de Vries, W., et al. 2006, ApJ, 647, 1040

Ebneter, K. A. 1989, Ph.D. Thesis California Univ. Berkeley

Ekers, R. D., Fanti, R., \& Miley, G. K. 1983, A\&A, 120, 297

Giovannini, G., Feretti, L., \& Comoretto, G. 1990 ApJ, 358, 159

Giovannini, G., Cotton, W. D., Feretti, L., Lara, L., \& Venturi, T. 1998, ApJ, 493, 632

Giovannini, G., Taylor, G. B., Arbizzani, E., et al. 1999, ApJ, 522, 101

Giovannini, G., Cotton, W. D., Feretti, L., Lara, L., \& Venturi, T. 2001, ApJ, 552, 508

Giroletti, M., Giovannini, G., Feretti, L., et al. 2004, ApJ, 600, 127

Machalski, J. 1998, A\&AS, 128, 15

Marcha, M. J. M., Browne, I. W. A., Impey, C. D., \& Smith, P. S. 1996, MNRAS, 281,425

Mirabel, I. F., \& Rodriguez, L. F. 1994, Nature, 371, 46

Morganti, R., Ulrich, M.-H., \& Tadhunter, C. N. 1992, MNRAS, 254, 546

Taylor, G. B., \& Vermeulen, R. C. 1997, ApJ, 485, L9 